\title{
CORTICOTROPIN-RELEASING FACTOR: CO-EXPRESSION WITHIN DISTINCT SUBSETS OF OXYTOCIN-, VASOPRESSIN-, AND NEUROTENSIN-IMMUNOREACTIVE NEURONS IN THE HYPOTHALAMUS OF THE MALE RAT ${ }^{1}$
}

\author{
P. E. SAWCHENKO, ${ }^{2}$ L. W. SWANSON, AND W. W. VALE
}

The Salk Institute for Biological Studies and The Clayton Foundation for Research-California Division, La Jolla, California 92037

Received October 11, 1983; Revised November 28, 1983; Accepted November 28, 1983

\begin{abstract}
Two immunohistochemical methods that allow the concurrent localization of neuroactive substances within individual neurons have been used to identify, count, and chart the distribution of corticotropin-releasing factor (CRF)-immunoreactive cells in the paraventricular nucleus of the hypothalamus (PVH) that may also contain an additional peptide. In colchicine-treated male rats a moderate number of oxytocin-stained cells, localized primarily in a discrete, anterior part of the magnocellular division of the nucleus, was found also to stain positively for CRF. Similarly, oxytocin and CRF immunoreactivity were jointly expressed in magnocellular neurons distributed diffusely in the supraoptic nucleus. Smaller numbers of vasopressin- and neurotensin-stained neurons centered in specific parts of the parvocellular division of the PVH were stained with antisera against CRF. Possible mechanisms whereby the function of subsets of magnocellular and parvocellular neurosecretory neurons can be modulated differentially are discussed.
\end{abstract}

The recent isolation and characterization (Vale et al. 1981) of a 41-residue peptide that appears to correspond to the long-elusive corticotropin-releasing factor (CRF) has had obvious physiological and clinical implications. Antisera to the peptide have also provided morphologists with a marker for the group of parvocellular neurosecretory neurons that stimulates the release of ACTH and $\beta$-endorphin from the anterior lobe of the pituitary gland. Immunohistochemical studies on the distribution of CRF-stained neurons (e.g., Bloom et al., 1982; Bugnon et al., 1982; Olschowka et al., 1982; Cummings et al., 1983; Merchenthaler et al., 1983; Swanson et al., 1983) confirm recent functional work (e.g., Makara et al., 1981; Ixart et al., 1982; Antoni et al., 1983) suggesting that while the peptide is widely distributed in the central nervous system, the vast majority of cells delivering the

\footnotetext{
${ }^{1}$ This work was supported by Grants NS-16686, DA-0029, and AM26741 from the United States Public Health Service and was conducted in part by the Clayton Foundation for Research-California Division; P. E. S., L. W. S., and W. W. V. are Clayton Foundation Investigators. We wish to thank Ms. Donna Chin for preparing histological material, Ms. Pat Thomas for typing the manuscript, and Mr. Kris Trulock for photographic help. We are especially grateful to Dr. M. Brown for providing an antiserum against neurotensin, Dr. J. Rivier for synthetic peptides used in blocking experiments, and Dr. F. Vandesande and Dr. $K$. Dierick $x$ for antisera against oxytocin and vasopressin.

${ }^{2}$ To whom correspondence should be addressed.
}

hormone to portal vessels in the median eminence are localized in the paraventricular nucleus of the hypothalamus (PVH). This nucleus is best known for its role in the synthesis and delivery of the nonapeptide hormones oxytocin and vasopressin to the posterior lobe of the pituitary, although it is now clear that the PVH also contains separate and topographically distinct subpopulations of neurons that project to the neurohemal zone of the median eminence and to autonomic centers in the brainstem and spinal cord (see Swanson and Sawchenko, 1983). In a recent analysis of the distribution of CRFimmunoreactive neurons in the rat brain (Swanson et al., 1983), it was noted that stained cells in the PVH are localized primarily in discrete parts of the parvocellular division of the nucleus, where their distribution overlaps in part with that of several other neuropeptides, including neurotensin (Kahn et al., 1980). In addition, a smaller number of CRF-stained cells were found in parts of the magnocellular division of the PVH in which oxytocincontaining cells are known to be concentrated (Rhodes et al., 1981a; Sawchenko and Swanson, 1982). In view of the apparent overlap in the distribution of the neuropeptides, as well as a large body of evidence indicating that oxytocin and (especially) vasopressin may serve to modulate ACTH secretion (see Yates and Maran, 1974; Vale et al., 1983), we have examined the possibility that CRF is co-expressed along with other peptides in individual 
neurons of the PVH. Other recent analyses have failed to provide a consistent view of this problem; CRF-immunoreactive neurons in the magnocellular division of the PVH have been reported to contain either oxytocin (Burlet et al., 1983) or vasopressin (Roth et al., 1983a) or neither peptide (Bugnon et al., 1982; Antoni et al., 1983).

\section{Materials and Methods}

Tissue preparation and immunohistochemical procedure. Adult male albino rats of the Sprague-Dawley strain were used in all experiments. Most animals received a single injection of 50 to $100 \mu \mathrm{g}$ of colchicine into the lateral ventricle 48 to $72 \mathrm{hr}$ before perfusion to arrest axonal transport and thereby enhance the immunohistochemical staining of neuropeptides in cell bodies. This pretreatment is necessary to demonstrate maximal numbers of CRF- and neurotensin-immunoreactive neurons in the PVH. The animals were perfused with ice-cold $4 \%$ paraformaldehyde in a two-phase procedure in which the $\mathrm{pH}$ of the perfusate is varied (Sawchenko et al., 1982; Swanson et al., 1983). Up to five one-in-five series of 20 $\mu \mathrm{m}$ thick frozen sections through the PVH were saved and then prepared for indirect immunofluorescence staining of cells that cross-react with antisera against CRF, neurotensin, oxytocin, or vasopressin using a conventional method (Sawchenko and Swanson, 1981) based upon the localization of a primary antiserum raised in rabbits using an affinity-purified, fluorescein-conjugated goat anti-rabbit IgG. The distributions of labeled cells were mapped onto projection drawings made from adjacent series of sections counterstained with thionin, and the region around the PVH was photographed at $\times 100$ magnification using Ilford XP-l film. The reaction product was then eluted from the tissue with a variant of the method described by 'Tramu et al. (1978). In brief, the sections were rinsed in phosphate-buffered saline (PBS) and then placed in a freshly prepared solution of $0.15 \mathrm{M}$ $\mathrm{KMnO}_{4}$ and $0.01 \mathrm{~N} \mathrm{H}_{2} \mathrm{SO}_{4}$ for 30 to $60 \mathrm{sec}$. They were then transferred immediately to $0.5 \mathrm{M} \mathrm{NaHSO}$ until decolorized and were rinsed thoroughly $(3 \times 10 \mathrm{~min})$ in PBS. The sections were then reincubated in fluoresceinlabeled secondary antiserum, rinsed $(2 \times 10 \mathrm{~min})$ in PBS, and inspected. The presence of any immunoreactivity in these sections was taken as evidence that the elution procedure had been less than maximally effective, and the material was either re-eluted or discarded. Sections in which the elution procedure was judged to have been complete were then processed for the demonstration of a second neuropeptide using the protocol described above, except that the incubations were carried out on slide-mounted sections. The sections were then rephotographed. Fnlargements $(8 \times 10$ inches $)$ of the two sets of photographs were made, and comparisons were made by superimposing tracings of all clearly stained neurons in one set of photographs onto the other. In this way, maps showing the location of cells in PVH that contained $\mathrm{CRF}$ and/or oxytocin, CRF and/or vasopressin, and CRF and/or neurotensin were generated from the same tissue sections.

Complete and evenly spaced series of sections through the PVH, in which staining for both antigens was adequate and controls for effective elution were negative, were generated for the oxytocin-CRF comparison ( $n=$ $4)$, the vasopressin-CRF comparison $(n=3)$, and the neurotensin-CRF comparison $(n=3)$. Over 50 partial series, generated from 23 rats, provided confirmation of the basic results described below.

As an additional control for method specificity, eight animals were pretreated and perfused as described above, and small blocks of hypothalamic tissue were cut serially in sections 5 to $6 \mu \mathrm{m}$ thick. The members of pairs of individual sections at all levels of the PVH were stained individually for the presence of oxytocin and CRF, or vasopressin and $\mathrm{CRF}$, or neurotensin and $\mathrm{CRF}$. Comparisons of the location and morphology of stained neurons in adjoining pairs of sections were again made photographically.

Antisera and controls. Antisera against oxytocin and vasopressin were provided by Dr. F. Vandesande and Dr. K. Dierickx (University of Ghent, Belgium) and were cross-adsorbed in the solid phase against the heterologous antigen according to a procedure outlined clsewhere (Swaab and Pool, 1975). Specific staining with each antiserum was blocked when immunoprocessing was carried out using sera that had been pre-incubated with an excess ( $15 \mathrm{mg} \mathrm{ml}^{-1}$ ) of the homologous synthetic peptide and then diluted to the working concentration of $1: 2000$.

The antiserum against neurotensin was provided by Dr. M. R. Brown (Peptide Biology Laboratory, The Salk Institute) and was prepared against neurotensin conjugated with glutaraldehyde to thyroglobulin. This serum (N1l-1) is directed against the C-terminal portion of the molecule (Brown et al., 1978) and was used at a dilution of 1:4000. All staining in the hypothalamus was blocked by the addition of synthetic neurotensin $\left(15 \mathrm{mg} \mathrm{ml}^{-1}\right)$ to the serum.

To ensure the validity of our localization of CRFimmunoreactive neurons, three sera against CRF human $\alpha$-globulin conjugates were used. Two of these (C24 and C30) are directed against the $\mathrm{C}$ - and $\mathrm{N}$-terminal portions, respectively, of the ovine CRF molecule (Swanson et al., 1983; Vale et al., 1983). The third was prepared against a synthetic rat CRF (Rivier et al., 1983) conjugate. Each of these sera was used at a final dilution of 1:3000, and pre-incubation of each with $22 \mathrm{mg} \mathrm{m}^{-1}$ of the respective immunogen blocked specific staining of cells and fibers in the hypothalamus. All sera used for both normal immunohistochemistry and blocking experiments contained $10 \mathrm{mg} \mathrm{ml}^{-1}$ of the "carrier" protein used for immunization.

Finally, although there has been no suggestion from our previous radioimmunoassay (Vale et al., 1983) or immunohistochemical (Swanson et al., 1983; P. E. Sawchenko and L. W. Swanson, unpublished data) studies of significant cross-reactivity of our anti-CRF sera with any of the other three peptides of interest here, about half of our co-localization experiments were carried out using anti-CRF sera that were preabsorbed with $20 \mathrm{mg}$ $\mathrm{ml}^{-1}$ of neurotensin, oxytocin, or vasopressin and antisera against the three latter peptides that were preincubated with an equivalent concentration of synthetic (ovine or rat) CRF. 


\section{Results}

Each of the three anti-CRF sera yielded patterns of staining in the PVH that were qualitatively similar and that were not altered by preabsorption with any of the other peptides of interest here. Staining with anti-rat CRF was somewhat brighter than that with antisera directed against ovine CRF, although direct counts of the total number of stained cells in the PVH with each antiserum were almost identical (about 2000). Therefore, we shall refer to CRF-immunoreactive cells, without reference to the species from which the immunogen was derived. Similarly, pre-incubation of antisera against oxytocin, vasopressin, or neurotensin with an excess of either synthetic ovine or rat CRF did not appear to alter the pattern of staining (or co-staining) that was seen with these antisera.

The distribution of CRF-, oxytocin-, vasopressin-, and neurotensin-stained cells in the PVH of material subjected to the elution procedure was in all respects similar to that seen in normal material (Sawchenko and Swanson, 1982; Swanson et al., 1983; P. E. Sawchenko and L. W. Swanson, unpublished observations). In the best of our elution experiments, actual counts of immunoreactive cells also corresponded closely to those found normally. Typically, however, a 10 to $15 \%$ reduction in the number of cells counted in the PVH was evident in eluted material, so that our counts of cells that stained for CRF and an additional peptide may slightly underestimate the actual size of the populations in which co-localization occurs.

The analysis described below is based on the results of sequential double staining studies. Nevertheless, each of the basic patterns of co-localization was confirmed using separate antisera to stain adjacent thin sections.

Oxytocin-CRF comparison. Of the three combinations examined, the greatest number of cells in the PVH with possible neuropeptide co-localization was observed with antisera against oxytocin and CRF. Such doubly labeled cells were concentrated in a discrete, anterior part of the magnocellular division of the nucleus (Fig. 1 and $2 A$; see Swanson and Kuypers, 1980, for parcellation), which corresponds to the anterior commissural nucleus of $\mathrm{Pe}$ terson (1966). In four complete series through the PVH, an average of $83 \pm 10$ cells in this part of the nucleus were stained for both antigens (Table I). This number constitutes about $40 \%$ of the total number of oxytocinstained neurons in the anterior magnocellular part of the PVH (Sawchenko and Swanson, 1982). Smaller numbers of cells containing oxytocin and CRF immunoreactivity were found in the medial and posterior magnocellular (Fig. 3) parts of the nucleus, even though the latter contains by far the greatest number of oxytocinergic cells in the nucleus. Indeed, a clear rostrocaudal gradient was evident, with progressively fewer doubly labeled cells in more caudal parts of the magnocellular division of the PVH (see Fig. 2).

Some $29 \%$ of all cells with both oxytocin and CRF immunoreactivity were scattered throughout most parts of the parvocellular division of the PVH. However, doubly labeled cells were never seen in the dorsal parvocellular part of the nucleus, whose principal efferent projec- tion is to the spinal cord (Sawchenko and Swanson, 1981). It should also be noted that a significant number of cells in the parvocellular division that stained for oxytocin and CRF were large (20 to $25 \mu \mathrm{m}$ in diameter) and were localized in close proximity to magnocellular ccll groups. This finding raises the possibility that at least some of these represent displaced magnocellular neurosecretory neurons, a small fraction of which are known to be dispersed through the parvocellular division (Sherlock et al., 1975; Swanson and Kuypers, 1980).

Consistent with the indications that a subset of magnocellular oxytocinergic neurons also express CRF immunoreactivity was the identification of a small number of cells in the supraoptic nucleus (SO) that also appear to express both peptides (Fig. 4). CRF-stained neurons in the SO were not found in all experiments, and they tended to be stained somewhat less intensely than those found in the magnocellular division of the PVH. Typically, not more than two or three were seen at any given rostrocaudal level of the nucleus. However, CRF-stained neurons did tend to be localized in the dorsal part of the SO, where oxytocinergic cells are known to predominate (see Swanson and Sawchenko, 1983). Consistent with this, all experiments that yielded unequivocal results indicated that CRF coexists exclusively with oxytocin in magnocellular neurons of the SO.

Vasopressin-CRF comparison. The distribution of vasopressin and of CRF-stained neurons in representative sections through the PVH is shown in Figure 5. In contrast to the results with oxytocin and CRF co-staining, the magnocellular division was largely devoid of cells that stained for vasopressin and CRF, except for a few cells in the anterior magnocellular part of the nucleus (Table I). Indeed, in the posterior half of the PVH the topographic segregation of vasopressin and of CRFstained cells is quite pronounced, and few, if any, doubly labeled cells were apparent at these levels (Figs. 5, $D$ to $F$ and 6 ). Surprisingly, small numbers of cells were found in the anterior parvocellular part of the nucleus that reliably stained for both peptides (Fig. $5, A$ and $B$ ). It should be emphasized that the average number of cells in the PVH that contained vasopressin and CRF immunoreactivity $(33 \pm 9)$ constitutes only about $3 \%$ of the total number of vasopressinergic cells in the nucleus (Sawchenko and Swanson, 1982). As mentioned above, CRF-stained cells in the SO were never found to stain with our antiserum against vasopressin.

Neurotensin-CRF comparison. In agreement with previously published reports (e.g., Kahn et al., 1980), neurotensin-stained cells were localized primarily in the same (anterior and medial) parts of the parvocellular division of the $\mathrm{PVH}$ in which CRF-immunoreactive cells are known to be concentrated, although their distributions here are distinctive (compare Figs. 5 and 7). Accordingly, an average of about 100 cells on each side of the brain were stained for both neurotensin and CRF and were found almost exclusively in just these regions (Fig. 8, Table I). It is likely that these counts substantially underestimate the number of cells in which these two peptides coexist since both cell types are very densely clustered in the dorsal medial parvocellular part of the PVH, which may well have compromised our ability to 

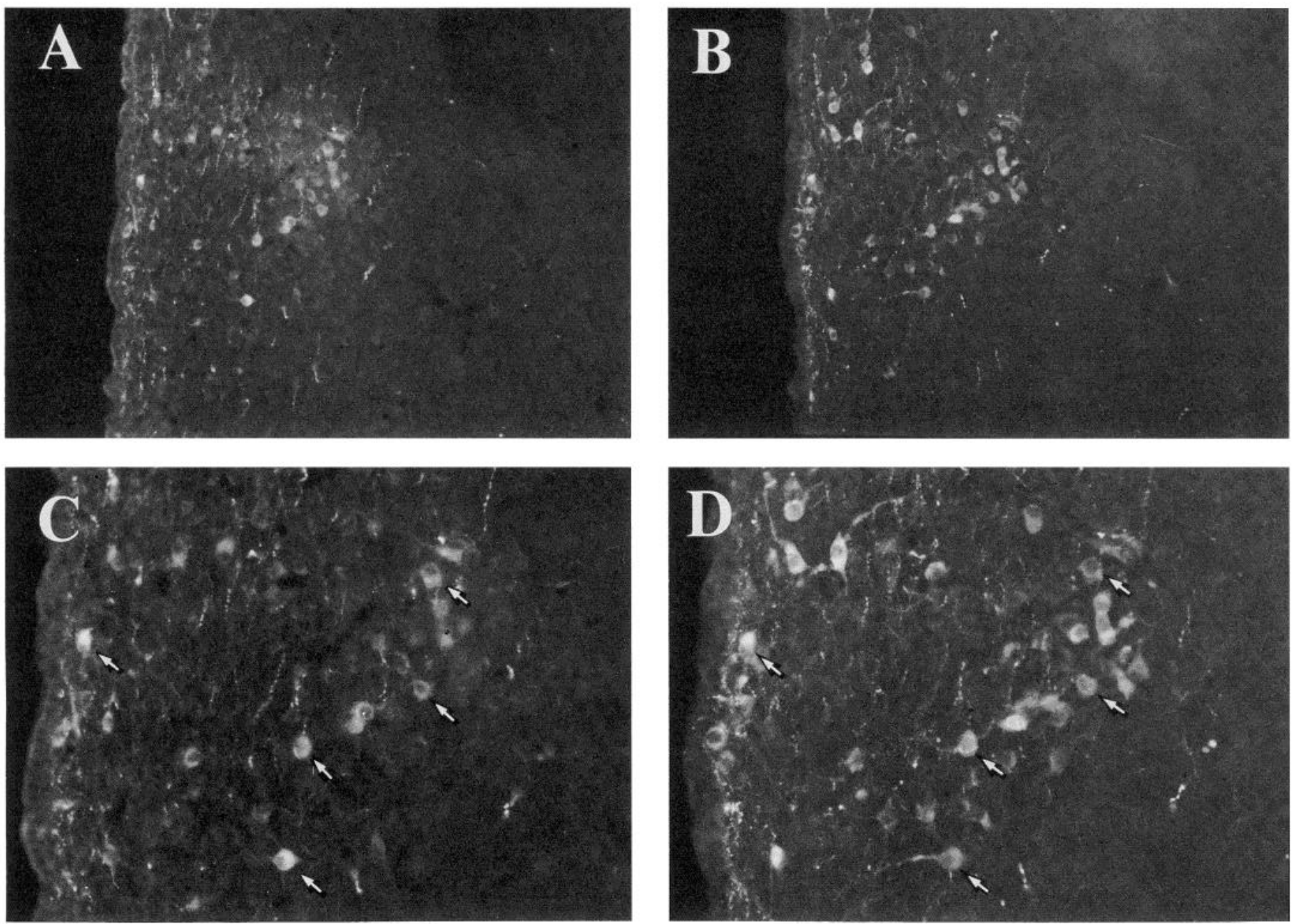

Figure 1. $A$ and $B$, Immunofluorescence photomicrographs of the anterior magnocellular part of the PVH to show the distribution of CRF- $(A)$ and oxytocin- $(B)$ immunoreactive cells in the same tissue section from a colchicine-pretreated rat. After the section was stained with an antiserum against CRF, the immunoreagents were eluted, and the tissue was restained with anti-oxytocin. The third ventricle is to the left; the descending column of the fornix is visible at the upper right. Magnification $\times 150$. $C$ and $D$, Higher magnification view of the same section to show that a significant proportion of magnocellular oxytocinstained cells at this level also express CRF immunoreactivity. Five cells that are clearly stained with both antisera are indicated (arrows). However, note that some cells in this field clearly express oxytocin or CRF immunoreactivity, but not both. Magnification $\times 275$.

identify additional doubly stained cells. One major difference in the distribution of these cell types is that neurotensin-stained cells were rarely seen in the magnocellular division of the PVH (and none of these were also CRF-stained) and were never found in the SO.

\section{Discussion}

In these experiments, CRF immunoreactivity was localized in subpopulations of oxytocin-, vasopressin-, and neurotensin-stained neurons in the PVH of the adult male rat. The three populations that presumably contain two different peptides were found to differ in size, and each was concentrated in an anatomically distinct part of the nucleus. Thus, cells stained for both oxytocin and $\mathrm{CRF}$ were found primarily in the anterior part of the magnocellular division of the PVH, as well as in the SO, which contains only magnocellular cells. In contrast, the few cells that were found to express CRF and vasopressin immunoreactivity were centered in the anterior part of the parvocellular division of the PVH and were never seen in the SO. A substantial number of neurotensinstained cells, centered in the medial and anterior parts of the parvocellular division, also stained with antisera against CRF. The apparent co-expression of these neuropeptides in the PVH suggests ways in which the function of subsets of chemically specified populations of hypothalamic neurons can be modulated selectively. Before discussing these results in detail, however, it is important to consider factors that bear on their validity.

Although technically cumbersome, the sequential immunostaining method of Tramu et al. (1978) provides a useful way to co-localize peptide antigens in individual neurons when primary antisera raised in different species are not available. In our experience this method is far better suited for determining the overall distribution and the proportion of cells in which co-localization occurs, 


\section{Oxytocin $\circ$ Double *
CRF $\bullet$}
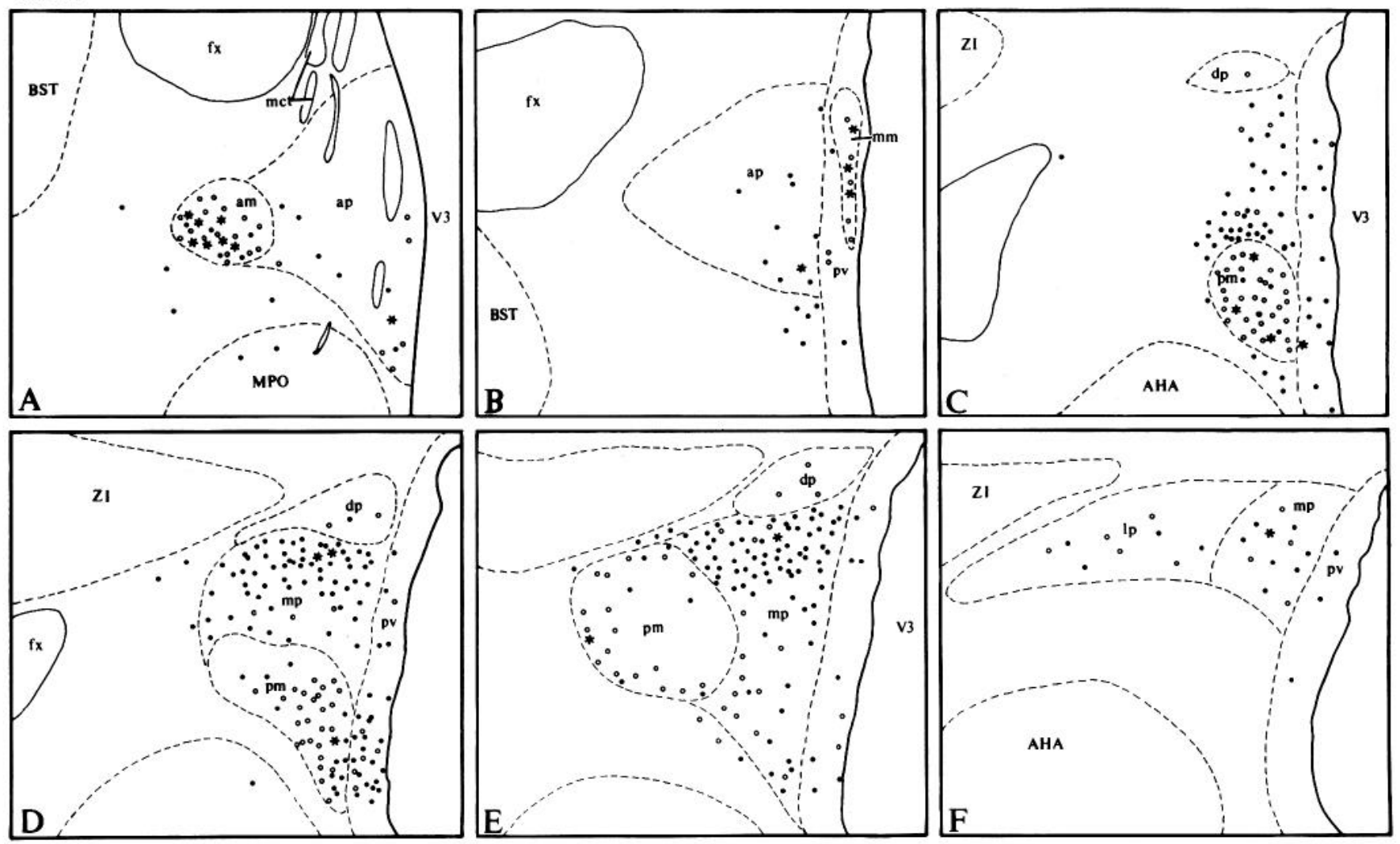

Figure 2. A series of line drawings through representative rostrocaudal $(A$ to $F$ ) levels of the $\mathrm{PVH}$ to show, in a single series of sections, the distribution of cells that stained with antisera against oxytocin (open circles), ovine CRF (solid circles), or both (asterisks). Antisera were differentially cross-absorbed against the heterologous peptide. Note that the doubly labeled cells are concentrated in rostral parts of the magnocellular division of the nucleus.
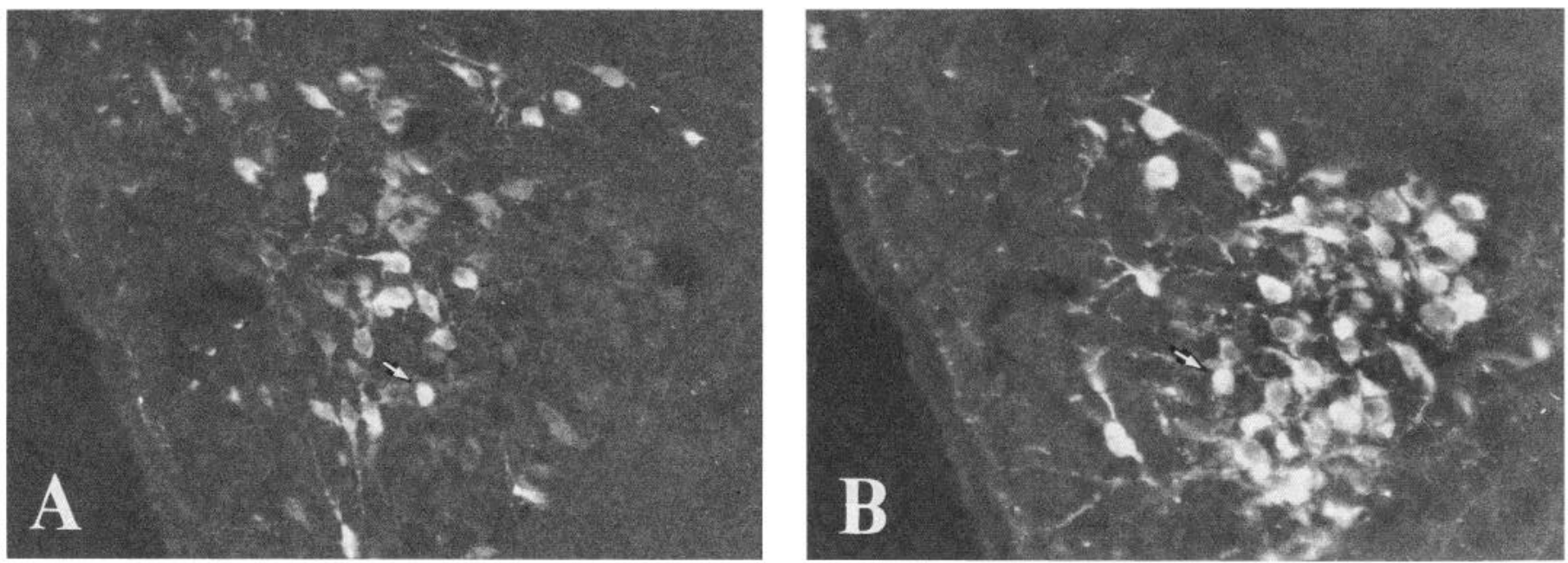

Figure 3. Fluorescence photomicrographs through the rostral zone of the posterior magnocellular part of the PVH (at approximately the level illustrated in Fig. $2 C$ ) to show the distribution of CRF $(A)$ and oxytocin $(B)$ immunoreactivity in a single section. In contrast to the anterior magnocellular part of the nucleus, CRF- and oxytocin-stained cells at this level are more strictly segregated in discrete parts of the parvocellular and magnocellular divisions of the PVH, respectively. Note also the difference in cell size. Only one neuron stained with both antisera (arrow) was found at this level. Colchicine-pretreated rat. Magnification $\times 275$. 
than is the comparison of staining patterns in adjacent thin sections. Nevertheless, each of the principal findings reported here was confirmed using the latter approach.

The validity of any immunohistochemical method depends upon the specificity of the antisera employed, and several points relevant to this consideration are worthy of comment. First, each of the results reported here was obtained with antisera that were cross-absorbed with concentrations of synthetic peptides in excess of those required to eliminate staining completely when added to the homologous antiserum. Second, antisera against oxytocin and vasopressin were differentially cross-adsorbed against one another, and the now well recognized differences in the distribution of oxytocin and vasopressin immunoreactivity in the rat hypothalamus (see Swanson and Sawchenko, 1983) provided histological confirmation of the effectiveness of this procedure. Finally, and perhaps most importantly, similar results were obtained using antisera against ovine $\mathrm{CRF}$, which have been shown in radioimmunoassay to recognize determinants on different regions of the CRF molecule, and using an additional antiserum against the recently characterized rat CRF molecule (Rivier et al., 1983). This makes it highly unlikely that our results can be attributed to spurious cross-reactivity of any single anti-CRF serum with an amino acid sequence in an unrelated molecule, or with a precursor form of oxytocin, vasopressin, or neurotensin.

Previous studies addressing the question of whether CRF may be expressed along with oxytocin or vasopressin in magnocellular neurosecretory neurons have provided conflicting results. Based on comparisons of immunohistochemical staining patterns, it has been suggested that $\mathrm{CRF}$ is not contained within oxytocinergic or vasopressinergic cells in the PVH (Bugnon et al., 1982; Antoni et al., 1983), although in one of these studies an "accessory paraventricular nucleus" was said to contain some CRF-immunoreactive cells, as was the SO (Bugnon et al., 1982). Approaching the issue directly, Roth et al. (1983a) described co-localization of CRF and vasopressin immunoreactivity in a subpopulation of magnocellular neurons in the PVH, but not in the SO, of colchicine-treated adrenalectomized rats. This result was not obtained in the Brattleboro rat, a mutant strain that is genetically unable to synthesize vasopressin. Most recently Burlet et al. (1983) provided results that are

TABLE I

Mean ( \pm SEM) number of cells containing immunoreactivity for two neuropeptides in subdivisions ${ }^{a}$ of the paraventricular nucleus on one side of the brain

\begin{tabular}{|c|c|c|c|c|c|c|c|c|c|}
\hline \multirow{2}{*}{ Antigens } & \multicolumn{5}{|c|}{ Parvocellular Division } & \multicolumn{3}{|c|}{ Magnocellular Division } & \multirow{2}{*}{ Total } \\
\hline & $\mathrm{AP}^{b}$ & PV & MP & $\mathrm{DP}$ & LP & $\mathrm{AM}$ & $\mathrm{MM}$ & $\mathrm{PM}$ & \\
\hline $\begin{array}{l}\text { Oxytocin-CRF } \\
\quad(n=4)\end{array}$ & $13 \pm 8$ & $20 \pm 11$ & $20 \pm 7$ & $0 \pm 0$ & $4 \pm 4$ & $83 \pm 10$ & $18 \pm 8$ & $36 \pm 12$ & $194 \pm 48$ \\
\hline $\begin{array}{l}\text { Vasopressin-CRF } \\
\quad(n=3)\end{array}$ & $18 \pm 10$ & $0 \pm 0$ & $4 \pm 4$ & $0 \pm 0$ & $0 \pm 0$ & $8 \pm 5$ & $0 \pm 0$ & $3 \pm 2$ & $33 \pm 9$ \\
\hline $\begin{array}{l}\text { Neurotensin-CRF } \\
\quad(n=3)\end{array}$ & $20 \pm 8$ & $6 \pm 2$ & $76 \pm 23$ & $0 \pm 0$ & $0 \pm 0$ & $0 \pm 0$ & $0 \pm 0$ & $0 \pm 0$ & $102 \pm 21$ \\
\hline
\end{tabular}

${ }^{a}$ For a description of the parcellation of the PVH, see Swanson and Kuypers (1980).

${ }^{b} \mathrm{AP}$, anterior parvocellular; PV, periventricular; MP, medial parvocellular; DP, dorsal parvocellular; LP, lateral parvocellular; AM, anterior magnocellular; MM, medial magnocellular; PM, posterior magnocellular.
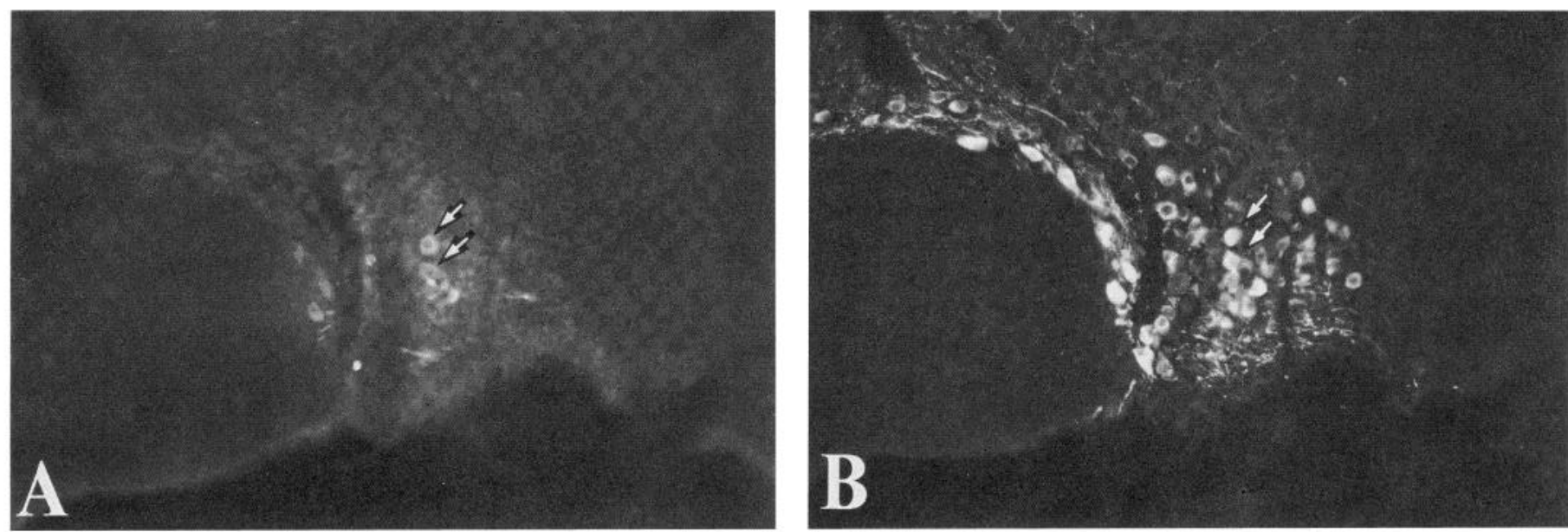

Figure 4. Fluorescence photomicrographs of a single section through roughly the mid-rostrocaudal extent of the SO to show the distribution of CRF- $(A)$ and oxytocin- $(B)$ stained neurons. Two cells (arrows) in this section are clearly stained with both antisera. CRF-stained cells were never found to stain with an antiserum against vasopressin. The optic chiasm is to the left. Colchicine-pretreated rat. Magnification $\times 150$. 
Vasopressin 。

\section{CRF •}

\section{Double *}
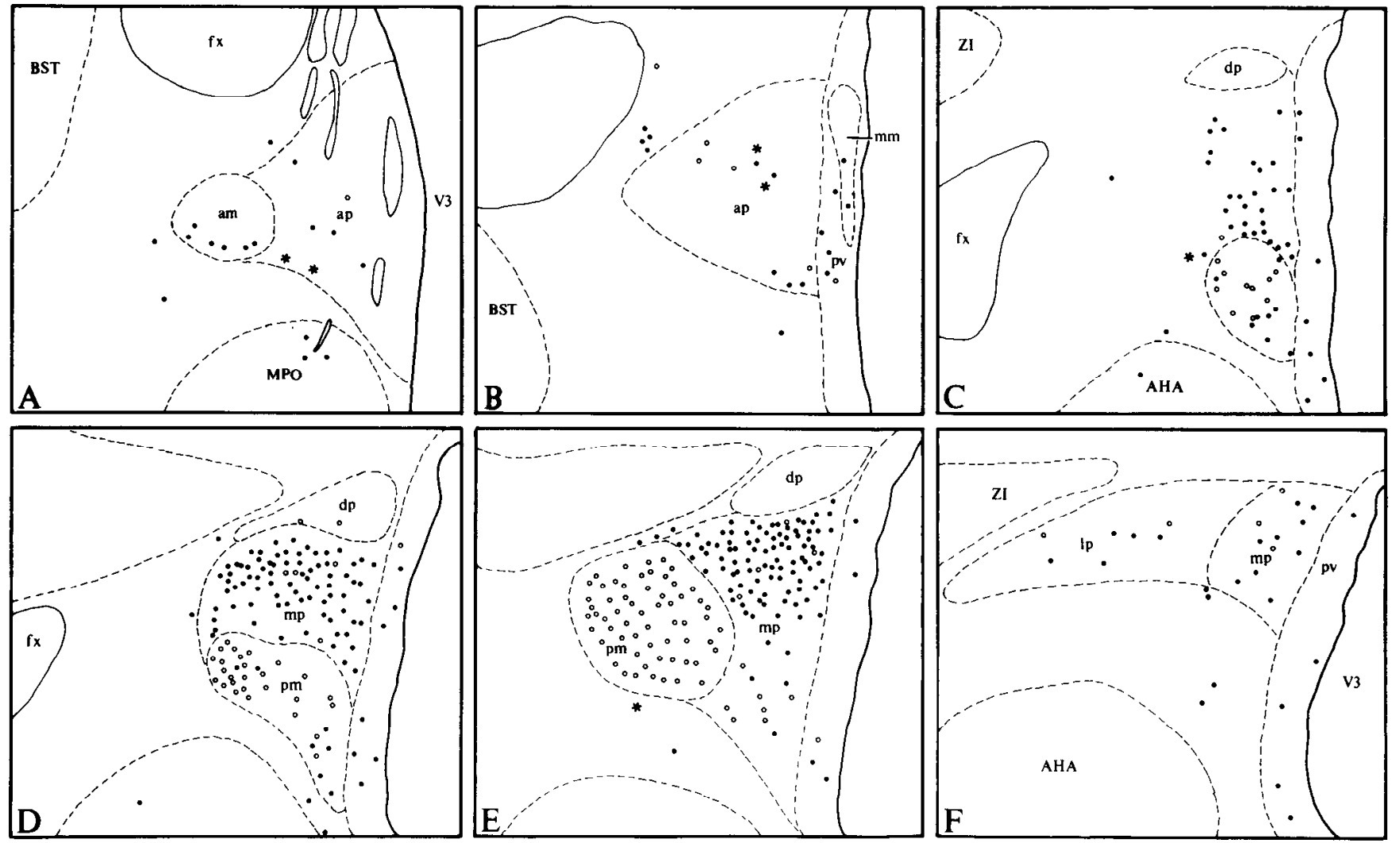

Figure 5. A series of line drawings through the rostrocaudal extent of the PVH to show the distribution of cells stained with antisera against vasopressin (open circles), CRF (solid circles), or both (asterisks). Very few cells were found to display both vasopressin and CRF immunoreactivity, and these were concentrated in the anterior part of the parvocellular division of the nucleus $(A$ and $B$ ). Note the rather strict topographic segregation of the two cell types through the caudal half of the nucleus.

generally compatible with those of the present study. These authors found that CRF and oxytocin immunoreactivity was expressed in a subset of rostrally situated magnocellular neurons in the region of the PVH. In the SO, however, only the most caudal (retrochiasmatic) part of the nucleus was found to contain cells that expressed both peptides. Similar results were reported in the Brattleboro rat, strengthening the conclusion that oxytocinergic cells are preferentially, if not exclusively, involved.

Several considerations raised by the present results, and some recent additional observations, may help to resolve these discrepancies. First, the localization of doubly stained cells in the anterior magnocellular part of the PVH strongly supports the conclusion that CRF immunoreactivity is preferentially associated with oxytocinergic magnocellular neurons, since two independent analyses confirm that the vast majority of magnocellular neurons here are oxytocinergic (Rhodes et al., 1981a; Sawchenko and Swanson, 1982). On the bases of contiguity and connectivity, we have considered this cell group as a part of the PVH (see Swanson and Sawchenko, 1983), although others have considered it as one of the larger "accessory" neurosecretory cell groups dispersed throughout the hypothalamus (Peterson, 1966; Bugnon et al., 1983). This may explain the failure of several groups to describe CRF-oxytocin co-localization in the magnocellular division of the PVH. In the caudal half of the nucleus, i.e., the posterior magnocellular part of Swanson and Kuypers (1980), very few cells were found jointly to express oxytocin and CRF immunoreactivity. Second, in considering the report that CRF immunoreactivity is localized in a subpopulation of vasopressinergic neurons (Roth et al., 1983a), it should be borne in mind that these results were obtained in animals that were pretreated with colchicine and adrenalectomized. Adrenalectomy has been shown to enhance CRF immunostaining in a subset of neurons centered in the parvocellular division of the PVH (e.g., Bugnon et al., 1983; Merchenthaler et al., 1983; Swanson et al., 1983), presumably as a consequence of the removal of circulating adrenal steroids, which provide a feedback inhibition of CRF production (see Yates and Maran, 1974). The very small population of neurons in the parvocellular division of the PVH that was found here to express both $\mathrm{CRF}$ and vasopressin immunoreactivity in animals pretreated only with colchicine seems unlikely to account for the findings of Roth et al. (1983a). However, we have recently found that after adrenalectomy alone, vasopressin immunoreactivity is expressed in a large subpopulation of CRF-stained neurons in the parvocellular division of the PVH (Sawchenko et al., 1984). The seemingly discrepant results of Roth et al. (1983a) may, therefore, 
be attributable to the use of two pretreatment regimens to enhance CRF immunostaining, one of which also appears to exert an effect on the expression of vasopressin immunoreactivity in the PVH. Finally, there are conflicting reports as to whether CRF is produced at all by neurons in the SO. Several laboratories have reported results compatible with those described here, namely, that a few weakly stained CRF cells may be seen in frontal sections through all levels of the $\mathrm{SO}$ in the rat (Bugnon et al., 1982; Olschowka et al., 1982; Cummings et al., 1983). Perhaps a relatively low level of CRF synthesis in this population is responsible for the failure of other groups (Antoni et al., 1983; Roth et al., 1983a), including our own (Swanson et al., 1983), to detect CRF immunoreactivity in these neurons or to detect it only in a topographically specified subpopulation (Burlet et al., 1983). However, it is of interest that in a number of other species, CRF-stained neurons in the SO appear to represent a sizable subpopulation and to be intensely stained (Kawata et al., 1982). The present report and
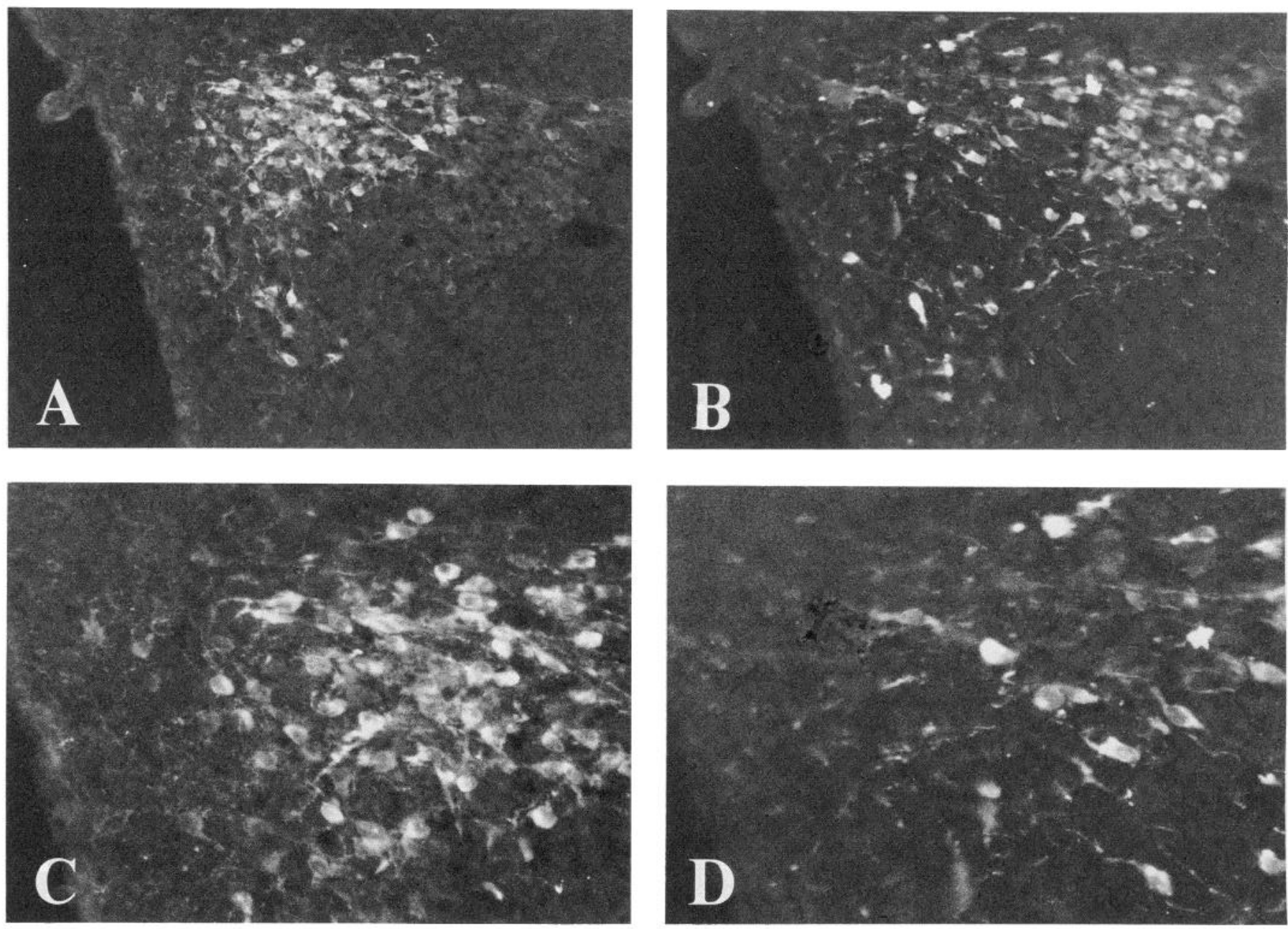

Figure 6. $A$ and $B$, Fluorescence photomicrographs of a section through a mid-rostrocaudal level of the PVH (at approximately the level illustrated in Fig. $5 E$ ) to show the distribution of CRF- $(A)$ and of vasopressin- $(B)$ immunoreactive neurons. Note an almost complete segregation of the two cell types. Colchicine-pretreated rat. Magnification $\times 150$. $C$ and $D$, Higher power photomicrographs through the medial parvocellular part of the PVH, where CRF-stained neurons $(C)$ are concentrated, and a few vasopressin-stained $(D)$ cells are seen. Even in this region of maximum overlap of the two distributions, no doubly labeled cells can be detected. Magnification $\times 275$. 


\section{Neurotensin $\circ$ Double *
CRF•}
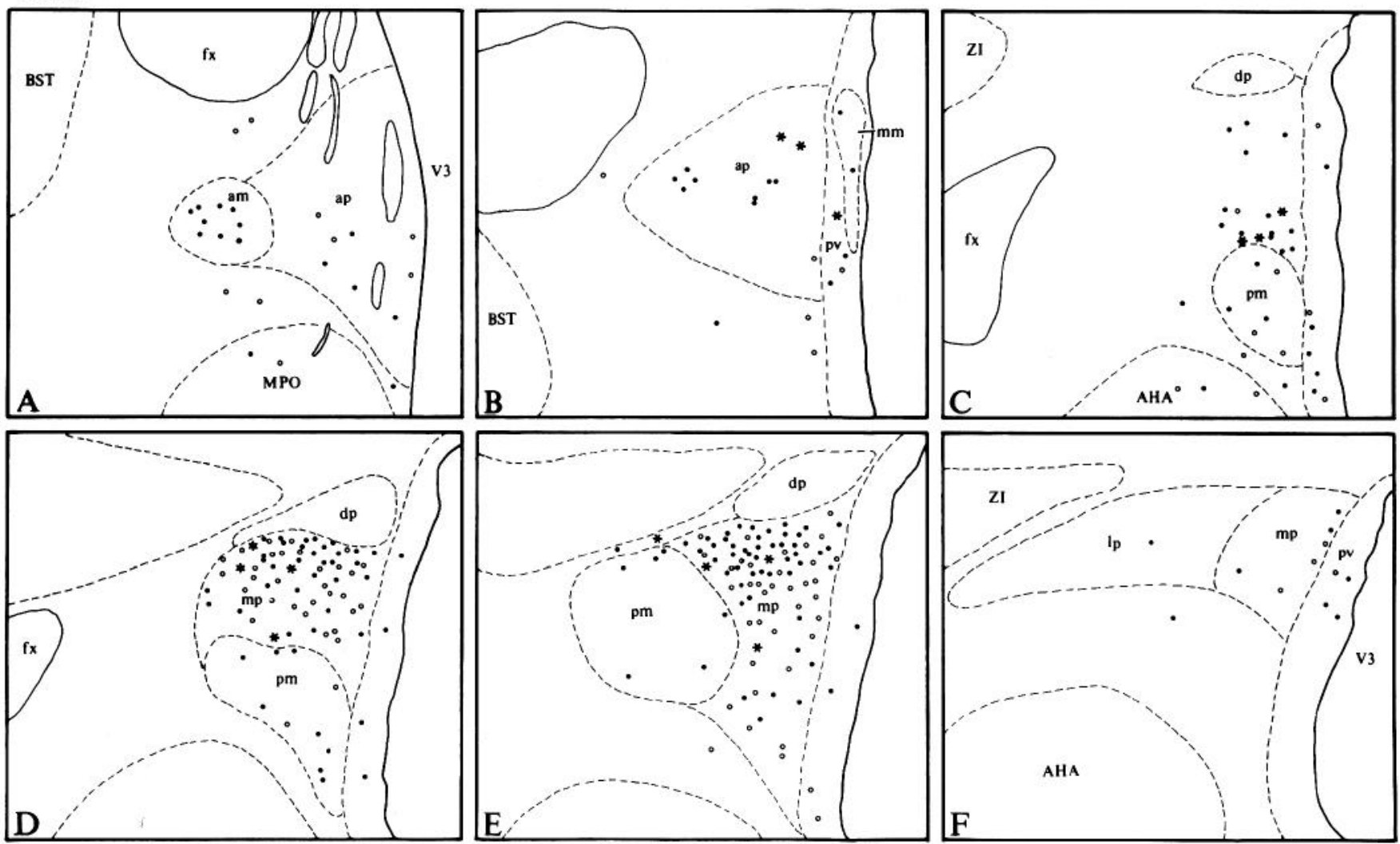

Figure 7. A series of line drawings through the rostrocaudal $(A$ to $F)$ extent of the PVH to compare the distribution of cells stained with antisera against neurotensin (open circles), CRF (solid circles), or both (asterisk), in representative sections from a single experiment. In contrast to the distribution of cells stained with anti-oxytocin or anti-vasopressin, that of neurotensinimmunoreactive neurons overlaps in part with that of CRF-stained cells throughout much of the parvocellular division, and both antigens were detected in a substantial number of cells in these regions.
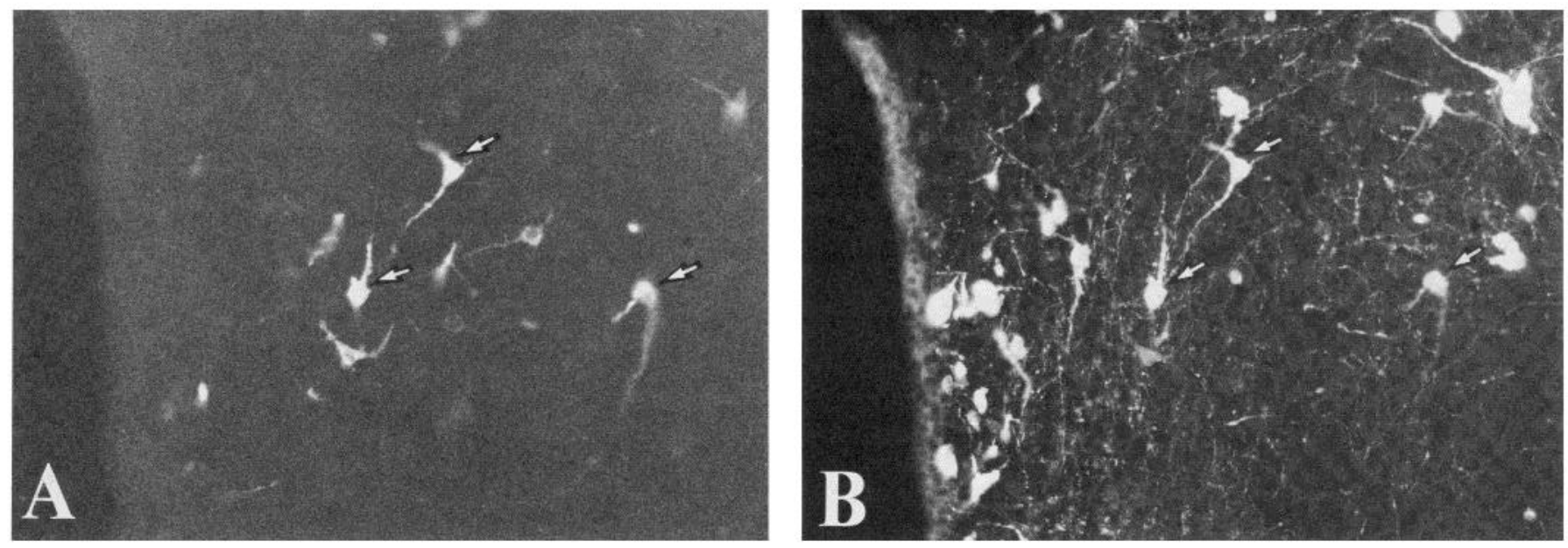

Figure 8. Fluorescence photomicrographs to show the distribution of CRF $(A)$ and neurotensin $(B)$ immunoreactivity in a single section through the anterior parvocellular part of the PVH (at approximately the level illustrated in Fig. $7 B$ ). Staining for neurotensin preceded that for CRF in this experiment. Both types of immunoreactivity are clearly present in three cells (arrows). Note that singly labeled cells of each type are readily apparent. Colchicine-pretreated rat. Magnification $\times 275$. 
neurons in the PVH and SO also appears to overlap with that of oxytocinergic cells (compare Watson et al., 1982; Khachaturian et al., 1982 with Rhodes et al., 1981a; Sawchenko and Swanson, 1982), and this question is currently being re-examined in our laboratory. This issue notwithstanding, our findings regarding the co-localization of CRF and oxytocin immunoreactivity differ somewhat from other reports of peptide coexistence in the magnocellular neurosecretory system in that a relatively small subset of oxytocinergic neurons appears to be selectively involved.

The functional significance of peptide co-localization in the magnocellular neurosecretory system is not yet clear. However, the presence of CRF in magnocellular neurons does suggest that the peptide may gain access to the general circulation. CRF-stained terminals have been reported in the posterior lobe of the pituitary gland (e.g., Bloom et al., 1982; Burlet et al., 1983), although it has been difficult to detect the peptides in peripheral blood using radioimmunoassay (Gibbs and Vale, 1982). $\mathrm{CRF}$ in magnocellular neurons may serve to modify the release of the neurohypophyseal peptide from the posterior lobe, or it may exert physiological effects at specific target tissues in the periphery. It is interesting to note that an effect of systemically administered CRF on a physiological parameter (blood pressure) has been reported that is opposite in sign to that seen when the peptide is injected centrally (Brown et al., 1982; Fisher et al., 1982). Thus, it will be of interest to determine whether the expression of CRF and oxytocin immunoreactivity in magnocellular neurons is regulated similarly and whether the anterior magnocellular part of the PVH receives a different complement of neural or blood-borne inputs than the remaining oxytocinergic cell groups. Thus far, a light microscopic analysis of the organization of afferent projections to the PVH and SO has provided little evidence for differential neural control (see Swanson and Sawchenko, 1983; Sawchenko and Swanson, 1983 for reviews). With respect to humoral factors, however, evidence suggestive of a differential control of magnocellular cell groups is available. Using a quantitative immunohistochemical method, Rhodes et al. (1981b) found that water deprivation and manipulation of gonadal hormone levels differentially influenced the oxytocin content of cells in different magnocellular cell groups, with the anterior magnocellular part of the $\mathrm{PVH}^{3}$ being most consistently affected.

Our finding that vasopressin and neurotensin immunoreactivities are expressed in discrete subsets of parvocellular CRF-stained neurons is similar to the pattern of partially overlapping distributions in the magnocellular system. This contrasts with the recent findings of Hokfelt et al. (1983), who described extensive overlap in the distribution of CRF-stained cells in the parvocellular division of the PVH with that of cells stained with antisera against both enkephalin and PHI. Vasopressin

\footnotetext{
${ }^{3}$ The anterior magnocellular and posterior magnocellular parts of the PVII are referred to in the study of Rhodes et al. (198b) as the anterior commissural nucleus and the paraventricular nucleus, respectively.
}

was once thought by some to be the principal CRF of the hypothalamus (see Yates and Maran, 1974), although it is now clear that the nonapeptide serves primarily to modulate the activity of the 41-residue peptide isolated and characterized by Vale et al. (1981). The present results, coupled with our recent finding that vasopressin immunoreactivity is expressed by a majority of parvocellular CRF-stained neurons in the adrenalectomized rat (Sawchenko et al., 1984), indicates that the two peptides which act synergistically to control the release of $\mathrm{ACTH}$ and $\beta$-endorphin from the anterior pituitary are produced by a single population of neurons. The significance of the observation that only a small fraction of these were detected in colchicine-treated, nonadrenalectomized animals is not yet clear.

Neurotensin-stained neurons were also found to have a distinctive localization in the parvocellular division of the PVH (see also Kahn et al., 1980), and some of these were also found to express CRF immunoreactivity. Neurotensin-stained fibers have been observed in the neurohemal zone of the median eminence (Kahn et al., 1980; Jennes et al., 1982), and effects of large doses of neurotensin on anterior pituitary hormone release have been described (e.g., McCann et al., 1982). Such effects have not yet been shown to include an influence on ACTH release.

In summary, we have described a partial correspondence in the distribution of CRF immunoreactivity with that of oxytocinergic magnocellular neurosecretory neurons, and with presumed parvocellular neurosecretory neurons that express either vasopressin or neurotensin immunoreactivity. Such distributions indicate that at least one functional consequence of neuropeptide coexistence in the PVH and SO may be to define subpopulations of neurons that express a given combination of peptides whose action in a given terminal field or target tissue may be different than that of either single peptide alone. Possible roles for co-existing peptides as local, autocrine or paracrine, regulatory agents at the level of the hypothalamus, median eminence, and pituitary must also be considered. Perhaps the most interesting possibility raised by these findings is that the synthesis of colocalized peptides on a neuron may change over the course of time as a function of its neural and endocrine inputs.

\section{References}

Antoni, F. A., M. Palkovits, G. B. Makara, E. A. Linton, P. J. Lowry, and J. Z. Kiss (1983) Immunoreactive corticotropinreleasing hormone in the hypothalamoinfundibular tract. Neuroendocrinology 36: 415-423.

Bloom, F. E., E. L. F. Battenberg, J. Rivier, and W. Vale (1982) Corticotropin releasing factor (CRF) immunoreactive neurons and fibers in rat hypothalamus. Reg. Peptides 4: 43-48.

Brown, M., J. Rivier, R. Kobayashi, and W. Vale (1978) Neurotensin-like and bombesin-like peptides: CNS distribution and action. In Gut Hormones, S. R. Bloom, ed., pp. 550-558, Churchill Livingston, Edinburgh.

Brown, M., L. A. Fisher, J. Rivier, J. Speiss, C. Rivier, and W. Vale (1982) Corticotropin releasing factor: Effects on the sympathetic nervous system and oxygen consumption. Life Sci. 30: 207-210. 
Bugnon, C., D. Fellman, A. Gouget, and J. Cardot (1982) Corticoliberin in rat brain: Immunocytochemical identification and localization of a novel neuroglandular system. Neurosci. Lett. 30: $25-30$.

Bugnon, C., D. Fellman, and A. Gouget (1983) Changes in corticoliberin and vasopressin-like immunoreactivities in the zona externa of the median eminence in adrenalectomized rats. Immunocytochemical study. Neurosci. Lett. 37: 43-49.

Burlet, A., M. -C. Tonon, P., Tankosic, D. Coy, and H. Vaudry (1983) Comparative immunocytochemical localization of corticotropin releasing factor (CRF-41) and neurohypophyseal peptides in the brain of Brattleboro and Long-Evans rats. Neuroendocrinology 37: 64-72.

Calza, L., K. Fuxe, L. F. Agnati, I. Zini, D. Ganten, R.E. Lang, K. Poulsen, and T. Hokfelt (1982) Presence of renin-like immunoreactivity in oxytocin immunoreactive nerve cells of the paraventricular and supraoptic nuclei in the rat hypothalamus. Acta Physiol. Scand. 116: 313-316.

Cummings, S., R. Flde, J. Fils, and A. Iindall (1983) Corticotropin-releasing factor immunoreactivity is widely distributed within the central nervous system of the rat: An immunohistochemical study. J. Neurosci. 3: 1355-1368.

Fisher, L. A., J. Rivier, C. Rivier, J. Speiss, W. Vale, and M. Brown (1982) Corticotropin releasing factor (CRF): Central effects on mean arterial pressure and heart rate in rats. Endocrinology 110: 2222 2224.

Gibbs, D. M., and W. Vale (1982) Presence of corticotropin releasing factor-like immunoreactivity in hypophyseal portal blood. Endocrinology 111: 1418-1420.

Hokfelt, T., J. Fahrenkrug, K. Takemoto, V. Mutt, S. Werner, A. -L. Hultings, L. Terenius, and K. J. Chang (1983) The PHI (PHI-27)/corticotropin-releasing factor/enkephalin immunoreactive hypothalamic neuron: Possible morphological basis for integrated control of prolactin, corticotropin, and growth hormone secretion. Proc. Natl. Acad. Sci. U. S. A. 80: $895-898$

Ixart, G., G. Alonso, A. Szatarczyk, F. Malaval, J. NougierSoule, and I. Assenmacher (1982) Adrenocorticotropic regulations after bilateral lesions of the paraventricular or supraoptic nuclei and in Brattleboro rats. Neuroendocrinology 35: $270-276$.

Jennes, L., W. E. Stumpf, and P. Kalivas (1982) Neurotensin: Topographical distribution in rat brain by immunohistochemistry. J. Comp. Neurol. 210: 211-224.

Kahn, D., G. 'M. Abrams, E. A. Zimmerman, R. Carraway, and S. E. Leeman (1980) Neurotensin neurons in the rat hypothalamus: An immunohistochemical study. Endocrinology 107: 47-54.

Kawata, M., K. Hashimoto, J. Takahara, and Y. Sano (1982) Immunohistochemical demonstration of corticotropin releasing factor-containing neurons in the hypothalamus of mammals including primates. Anat. Embryol. 165: 303-313.

Khachaturian, H., S. J. Watson, M. E. Lewis, D. Coy, A. Goldstein, and H. Skil (1982) Dynorphin immunocytochemistry in the rat central nervous system. Peptides 3: 941-952.

Kilcoyne, M. M., D. L. Hoffman, and E. A. Zimmerman (1980) Immunocytochemical localization of angiotensin II and vasopressin in rat hypothalamus: Evidence for production in the same neuron. Clin. Sci. 59: 575-605.

Makara, G. B., E. Stark, M. Karteszi, M. Palkovitz, and G. Rappay (1981) Effects of paraventricular lesions on stimulated ACTH release and CRF in stalk-median eminence of the rat. Am. J. Physiol. 240: E441-446.

McCann, S. M., E. Vijayan, J. Koenig, and L. Krulich (1982) The effects of neurotensin on anterior pituitary hormone secretion. In Neurotensin, A Brain as Gastrointestinal Peptide, C. B. Nemeroff and A. J. Prange, Jr., eds., pp. 160-171, New York Academy of Sciences, New York.
Merchenthaler, I., S. Vigh, P. Petrusz, and A. V. Schally (1982) Immunocytochemical localization of corticotropin-releasing factor (CRF) in the rat brain. Am. J. Anat. 165: 385-396.

Merchenthaler, I., S. Vigh, P. Petrusz, and A. V. Shally (1983) The paraventriculo-infundibular corticotropin releasing factor (CRF) pathway as revealed by immunocytochemistry in long-term hypophysectomized or adrenalectomized rats. Reg. Peptides 5: 295-305.

Olschowka, J. A., 'T. L. O'Donohue, G. P. Mueller, and D. M. Jacobowitz (1982) The distribution of corticotropin releasing factor-like immunoreactive neurons in rat brain. Peptides 3 : 995-1015.

Peterson, R. P. (1966) Magnocellular neurosecretory centers in the rat hypothalamus. J. Comp. Neurol. 128: 181-190.

Rhodes, C. H., J. I. Morrell, and D. W. Pfaff (1981a) Immunohistochemical analysis of magnocellular elements in rat hypothalamus: Distribution and numbers of cells containing neurophysin, oxytocin, and vasopressin. J. Comp. Neurol. 198: 45-64

Rhodes, C. H., J. I. Morrell, and D. W. Pfaff (1981b) Changes in oxytocin content in magnocellular neurons of the rat hypothalamus following water deprivation or estrogen treatment. Cell Tissue Res. 216: 47-55.

Rivier, J., J. Speiss, and W. Vale (1983) Characterization of rat, hypothalamic corticotropin releasing factor. Proc. Natl. Acad. Sci. U. S. A. 80: 4851-4855.

Roth, K. A., E. Weber, and J. D. Barchas (1983a) Immunoreactive corticotropin releasing factor (CRF) and vasopressin are colocalized in a subpopulation of the immunoreactive vasopressin cells in the paraventricular nucleus of the hypothalamus. Life Sci. 31: 1857-1860.

Roth, K. A., E. Weber and J. D. Barchas (1983b) Immunoreactive dynorphin (1-8) and corticotropin releasing factor in subpopulation of hypothalamic neurons. Science 219: 189191.

Sawchenko, P. E., and L. W. Swanson (1981) A method for tracing biochemically defined pathways in the central nervous system using combined fluorescence retrograde transport and immunohistochemical techniques. Brain Res. 210: 3151 .

Sawchenko, P. E., and L. W. Swanson (1982) Immunohistochemical identification of neurons in the paraventricular nucleus of the hypothalamus that project to the medulla or to the spinal cord in the rat. J. Comp. Neurol. 205: 260-272.

Sawchenko, P. E., and L. W. Swanson (1983) The organization and biochemical specificity of afferent projections to the paraventricular and supraoptic nuclei. Prog. Brain Res. 60: 19-29.

Sawchenko, P. E., L. W. Swanson, and S. A. Joseph (1982) The distribution and cells of origin of ACTH (1-39)-stained varicosities in the paraventricular and supraoptic nuclei. Brain Res. 232: 365-374.

Sawchenka, P. E., L. W. Swanson, and W. W. Vale (1984) Coexpression of corticotropin-releasing factor and vasopressin immunoreactivity in parvocellular neurosecretory neurons of the adrenalectomized rat. Proc. Natl. Acad. Sci. U. S. A., in press.

Sherlock, D. A., P. M. Field, and G. Raisman (1975) Retrograde transport of horseradish peroxidase in the magnocellular neurosecretory system of the rat. BrainRes. 88: 403-414.

Swaab, D. F., and C. W. Pool (1975) Specificity of oxytocin and vasopressin immunofluorescence. J. Endocrinol. 66: 263272.

Swanson, L. W., and H. G. J. M. Kuypers (1980) The paraventricular nucleus of the hypothalamus: Cytoarchitectonic subdivisions and the organization projections to the pituitary, dorsal vagal complex and spinal cord as demonstrated by retrograde fluorescence labeling methods. J. Comp. Neurol. 
194: 555-570.

Swanson, L. W., and P. E. Sawchenko (1983) Hypothalamic integration: Organization of the paraventricular and supraoptic nuclei. Annu. Rev. Neurosci. 6: 275-325.

Swanson, L. W., P. E. Sawchenko, J. Rivier, and W. W. Vale (1983) Organization of ovine corticotropin-releasing factor immunoreactive cells and fibers in the rat brain: An immunohistochemical study. Neuroendocrinology 36: 165-186.

Tager, H., M. Hohenboken, J. Markese, and R. J. Dinerstein (1980) Identification and localization of glucagon-related peptides in rat brain. Proc. Natl. Acad. Sci. U. S. A. 77:62296233.

Tramu, G., A. Pillez, and J. Leonardelli (1978) An efficient method of antibody elution for the successive or simultaneous localization of two antigens by immunohistochemistry. J. Histochem. Cytochem. 26: 322-324.

Vale, W. W., C. Rivier, and M. Brown (1977) Regulatory peptides of the hypothalamus. Annu. Rev. Physiol. 39: 473527.
Vale, W., J. Speiss, C. Rivier, and J. Rivier (1981) Characterization of a 41-residue ovine hypothalamic peptide that stimulates secretion of corticotropin and $\beta$-endorphin. Science 213: 1394-1397.

Vale, W., J. Vaughan, G. Yamamoto, T. Bruhn, C. Douglas, D. Dalton, C. Rivier, and J. Rivier (1983) Assay of corticotropin releasing factor. Methods Enzymol. 103: 565-577.

Vanderhaeghen, J. J., F. Lotstra, F. Vandesande, and K. Dierickx (1981) Coexistence of cholecystokinin and oxytocinneurophysin in some hypothalamohypophyseal neurons. Cell Tissue Res. 221: 227-231.

Watson, S. J., H. Akil, W. Fischli, A. Goldstein, E. Zimmerman, G. Nilaver, and T. B. Van Wiersma Greidanus (1982) Dynorphin and vasopressin: Common localization in magnocellular neurons. Science 216: 85-87.

Yates, F. E., and J. W. Maran (1974) Stimulation and inhibition of adrenocorticotropin release. In Handbook of Physiology, E. Knobil and W. H. Sawyer, eds., Sect. 7, Vol. IV, Part 2, pp. 367-404, American Physiological Society, Washington, D.C. 